Fecha de recepción: febrero 2017 Fecha de aceptación: agosto 2017 Versión final: septiembre 2018

\section{Enfoques teóricos de diseño que propenden hacia el desarrollo sostenible de Latinoamérica}

Liliana Patricia Durán Bobadilla *

y Luis Daniel Mancipe Lopez ${ }^{\star *}$

Resumen: El texto presenta un estudio bibliométrico de documentos científicos (artículo, libro, tesis e informe de investigación) en los que se encuentran los principales enfoques teóricos del diseño, publicados en revistas indexadas latinoamericanas entre 2000 y 2016. Busca construir una revisión de las perspectivas predominantes relacionadas con el impacto de la disciplina en el desarrollo de comunidades. Se tomaron como base las categorías: pensamiento de diseño, diseño centrado en las personas-usuarios, co-creación y gestión estratégica del diseño (más las emergentes). Se concluye con un cuadro comparativo que sintetiza estos enfoques, permitiendo identificar antecedentes, que se constituyen como aportes a proyectos de investigación actuales y futuros.

Palabras clave: Innovación social - desarrollo sostenible - co-creación - gestión estratégica del diseño - territorio.

[Resúmenes en inglés y portugués en las páginas 192-193]

${ }^{(*)}$ Magíster en Diseño y Creación Interactiva de la Universidad de Caldas, Colombia. Diseñadora Gráfica de la Universidad Jorge Tadeo Lozano. Docente de investigación del programa Diseño Gráfico CUN Regional Tolima.

${ }^{(*)}$ Master en internacionalización del desarrollo local, de la Universidad de Bologna, Italia, Diseñador Industrial de la Universidad Nacional de Colombia. Docente de investigación del programa Diseño Gráfico CUN Regional Bogotá.

\title{
Introducción
}

La discusión sobre la naturaleza del objeto de estudio del diseño como disciplina ha sido nutrida pero finalmente inacabada. Muchos teóricos como Frascara y González Ruiz lo han relacionado con las teorías de la comunicación como campo, otros como Verganti exaltan la capacidad única del diseño en la creación de innovaciones de "significado", tomadas como una reinterpretación de cómo los usuarios piensan los objetos, las gráficas o los productos físicos de la actividad de diseñar. Sin embargo, a la luz de las múltiples teorías y enfoques mayormente europeos o norteamericanos, se encuentra un fuerte vacío teórico de cómo debe afrontarse la profesión en Latinoamérica. 
Las condiciones territoriales, históricas, sociológicas y de sistema económico que tienen los países latinoamericanos son particulares y diferentes a las de los del "primer mundo", haciendo que muchas veces los enfoques de diseño traídos por profesionales que han estudiado en escuelas de diseño alrededor del globo se sientan importados, prestados y posiblemente no pertinentes.

En los últimos años el diseño ha tomado un viraje interesante acercándose cada vez más a la innovación social y al desarrollo, y abandonando un poco el enfoque de los diseñadores vedette que producen objetos y gráficas exquisitas para un público selecto. Además, la forma de pensar y de trabajar de los diseñadores se ha transferido a las disciplinas de los negocios, debido a la forma única de la profesión de conectar las necesidades simbólicas o el sentir de la población, con las dinámicas industriales y productivas. El diseño centrado en el usuario, el pensamiento de diseño, el codiseño, la co-creación y gestión estratégica de diseño, han logrado conectar la disciplina con la innovación social en muchos puntos, y han demostrado que el diseño si es pertinente en nuestros contextos.

La intención de este estado del arte es la de realizar una evaluación sistemática de cuáles han sido los enfoques teóricos del diseño que tienen un énfasis en innovación social o impacto en las comunidades para así poder repensar la profesión en prospectiva. Así mismo, el artículo es parte de la primera fase del macroproyecto de investigación "La comunicación y el Diseño en escenarios y actores sociales con el enfoque sistémico del desarrollo sostenible", de la Escuela de Comunicación y Bellas Artes de la Corporación Unificada Nacional de Educación Superior (CUN), en donde uno de sus objetivos busca determinar un enfoque teórico sobre el rol del diseño como profesión hacia las comunidades productivas en Colombia, por lo cual se definió desarrollar un estado del arte sobre los enfoques teóricos de la relación innovación - diseño en latinoamérica que se relacionen con la innovación social e impacto en estos contextos. En ese sentido nos preguntamos: ¿Cuáles son los enfoques predominantes del diseño como disciplina en Latinoamérica que se relacionan con el desarrollo sostenible?

\section{Método}

Para la construcción del estado del arte según la temática propuesta, se utilizó la metodología de estudio bibliométrico, por medio de la ubicación de las palabras clave: Gestión estratégica de diseño, Innovación social, Design driven innovation (innovación dirigida por el diseño), User centered innovation (innovación centrada por el usuario), Co-creación - co-diseño, Design thinking (pensamiento de diseño), en publicaciones y revistas indexadas latinoamericanas desde el año 2000 hasta el 2016 para la crear una base de datos como aporte a la caracterización de la acción estratégica del diseño en el contexto latinoamericano.

Por otra parte, se realizó el seguimiento bibliográfico de dichas publicaciones para enriquecer la información y el encuentro de posibles categorías emergentes. De estas se priorizaron aquellas que tuvieran un ranking relevante en el índice $H$ de Google Scholar y se sistematizaron leyendo el contenido de los artículos y sintetizando las posturas teóricas y metodológicas, lo cual permitió dar cuenta de las diferentes posturas teóricas y de esta 
manera, propender a la construcción de una línea de tiempo relacional con un ejercicio de visualización de datos.

\section{Resultados}

\subsection{Marco teórico-conceptual de referencia}

\subsubsection{La particularización de la disciplina del Diseño}

"El diseño es un plan mental y no tiene nada que ver con dibujar" (Ronald Shakespear)

Se ha debatido profundamente sobre el objeto del estudio del diseño, y finalmente esta discusión está inacabada. La diseñadora argentina Rosario Bernatene (2007) aporta a la cuestión la idea de que el diseño tiene diferentes enfoques históricos y todos ellos son válidos:

Tenemos un legado histórico para aquellos que desean profundizar en su rol emancipador-como se lo planteaban los manifiestos del arte concretoinvención-, otro para los que desean insistir en su función poética, otro para quienes quieren trabajar en su función democratizadora, o en su función fetiche, o como herramienta de desarrollo regional, como palanca para el fortalecimiento de las pymes, como interfaz entre ciencias básicas y tecnología de punta con la vida diaria, como acompañamiento de programas artesanales, o juntando varias de estas opciones. (p. 47)

Por otro lado, Julier (2010) afirma que se han superado las demarcaciones tradicionales dentro de las divisiones del diseño (gráfico, de modas, industrial, etc.) a una visión y estructuración de negocio. Esto podría significar una transición hacia una visión estratégica que abandona la simple idea del diseño como solucionador de problemas puntuales. Es así como en este artículo, se partirá sobre el diseño como un instrumento para el desarrollo sostenible y para esto es necesario acordar qué es diseño y qué es desarrollo.

\subsubsection{La relación entre el Diseño y la Comunicación en Latinoamérica}

Hablar del Diseño como disciplina abarca un terreno amplio y ambiguo si se carece de un enfoque determinado, es por eso, que para dar inicio a los antecedentes se tomará como base a Uribe (2009) en su tesis de maestría en Diseño y Creación Interactiva de la Universidad de Caldas en Colombia, acerca de la caracterización del objeto de estudio del diseño de comunicación (gráfico / visual), en donde se identificó el concepto de la comunicación partiendo del latín Cum "con" o "junto a" y Unio de "unión”. Comunicare es la puesta en común o una unión entre uno y otro. (2009, p. 56); así mismo, Uribe definió que: el fenómeno de la comunicación en términos de compartir significados habla del uso de diversos códigos: "El código gráfico/visual, integrado a otros, empezó a ser estudiado desde la mirada comunicacional, de tal manera que alcanzó a hablarse de la Comunicación Gráfica” (2009, p. 53). Esto indica que uno de los enfoques teóricos del Diseño se asocia a la comunicación en aspectos sociales según la experiencia de individuos que reconocen signos en mensajes específicos. 
Por su parte, Frascara (2006) presentó una definición de Diseño Gráfico aprobada por la International Council of Graphic Design Associations - ICOGRADA que se refiere al "Diseño de Comunicación Visual" dotada de tres elementos que integran dicho campo de actividad, 1. Tiene un método que es el Diseño, 2. Un objetivo, la Comunicación; y 3. Un medio, la Visión. En ese sentido, Frascara dice que: "el diseño de comunicación visual, visto como una actividad, es la acción de concebir, programar y realizar comunicaciones visuales, producidas en general por medios industriales y destinadas a transmitir mensajes específicos a grupos determinados" (2006, p. 25). En ese sentido, el punto de vista de Jorge Frascara en Latinoamérica se identifica dentro de los canales de información que de manera activa evolucionan con la tecnología que se vive en el siglo XXI, esto, sumado a la habilidad del diseñador que concibe el diseño como mediador del proceso de comunicación. Así mismo, González Ruiz (1994) describió la comunicación visual como el lenguaje de la visión, el cual enmarca todas las experiencias que establecen un diálogo entre el emisor y el receptor del mensaje que puede tener caminos impresos o audiovisuales de manera que: “dentro de ese contexto de la comunicación visual se inserta el Diseño Gráfico" y definió al Diseño Gráfico como: "la disciplina que posibilita comunicar visualmente, ideas, hechos, y valores útiles para la vida y el bienestar del hombre mediante una actividad proyectual" (1994, p. 122). El aporte de González Ruiz evidencia el enfoque del diseñador entorno al medio que lo rodea debido a que cita al brasileño Joaquim Redig, embajador del diseño Latino al citar los cinco principios rectores del diseño: a) el hombre, b) la utilidad, c) el ambiente, d) la economía, e) la forma. (1994, p. 123)

Los enfoques teóricos del Diseño que propenden hacia el desarrollo sostenible en Latinoamérica, han llegado en principio de regiones Europeas, desde los aportes de las escuelas de Diseño en Alemania (la Bauhaus y la Escuela de Ulm), como la necesidad de comunicar las ideas a través de productos del diseño compuestos de orden, propósito, significado y valor hacia la sociedad, según exponentes del diseño proyectual como Bruce Archer (1922-2005), es pertinente reconocer que:

El aporte de Archer determinó el camino de la formación disciplinar de los individuos quienes más allá de potencializar sus habilidades cognitivas y el manejo de principios del diseño, deben formarse en procesos metodológicos con los cuales pueden entender el entorno en que habitan, el contexto, identificar problemas frente a la información que requieren transmitir a través del diseño y las acciones de mejoramiento que se producen como resultado de la aplicación adecuada de la teoría y la práctica, de manera que proyecten al usuario en la experiencia de interpretar el significado presente en el diseño, de esta manera la educación de los diseñadores cobra sentido en las áreas de conocimiento descritas anteriormente. (Duran, 2015, p. 20)

Por otra parte, Horta Mesa (2004) aportó que para el diseño Latinoamericano, la experiencia europea postuló una idea de modernidad con la intención de dar respuesta a una tríada tradicional de problemas planteados históricamente por la arquitectura acerca de la forma, la función y la técnica, en ese sentido opinó que: 


\begin{abstract}
Mucho antes del modernismo latinoamericano, el arte y la cultura latinoamericana exhibía muestras de una concientización y un sólido pensamiento de aguda destreza y representación, que regentea el presente en muchos campos de la creación y la producción, y que por supuesto asimila el diseño. La importancia histórica realizada por la Bauhaus, justo sustantivó para el plano de la enseñanza del arte y el diseño un criterio de utilidad y democratización de la creación. (2004, p. 9)
\end{abstract}

Estas han sido pistas que conectan el pensamiento de diseño con la relación del entorno, los problemas sociales, políticos y económicos que a todo diseñador impactan desde las primeras escuelas de Diseño. Por otra parte, en relación con los enfoques europeos, se destaca a Manzini (2009), desde Italia, con el "nuevo conocimiento del Diseño" para la era contemporánea, el cual permite diferenciar espacios de encuentro hacia la conformación de grupos, comunidades, centros de investigación que fomenten el trabajo colaborativo, interdisciplinar en donde el diseñador pueda llevar una responsabilidad social con un pensamiento autónomo, permite tomar decisiones desde su reflexión como individuo frente al mundo que habita, proyectarse, investigar, crear estrategias en la formación del nuevo conocimiento.

El pensamiento de Manzini se relaciona con la observación del rol del diseñador en una comunidad encaminada a la solución de problemas en el campo del diseño sostenible, para identificar el individuo como punto de partida en una reflexión frente al comportamiento en el entorno que habita y esto se evidencia en todos los campos en los cuales actúa como ser humano, formando un sistema que permite el desarrollo del diario vivir.

\title{
2.1.3. Comunicación y desarrollo
}

Siendo amplia y difusa la discusión del diseño como disciplina, la del rol de la comunicación en el desarrollo lo es aún más. Partimos de que, el desarrollo es un proceso social, un consenso para la acción con el fin de mejorar el bienestar de una comunidad, teniendo en cuenta los intereses, las necesidades y las capacidades del territorio. El tipo y características del desarrollo están determinadas por el contexto y no existe una forma única de desarrollarse. Esta definición está construida a partir de varios documentos que dan un enfoque contemporáneo a esta palabra, superando la definición anacrónica desarrollada a partir de los años 50, la cual equiparaba desarrollo con modernización y determinaba que para que las poblaciones tuvieran un desarrollo simétrico debían cumplir unas condiciones únicas. Esto quiere decir que algunos países subdesarrollados debían adoptar dinámicas e imitar los valores de los países desarrollados desde un enfoque economicista para poder mejorar su bienestar (Beltrán, 2006; Dissanayake, 2006). En este orden de ideas, la comunicación debía ser una herramienta de difusión para el cambio de comportamiento de estas sociedades “ignorantes". Este enfoque fue bastante polémico, pues tiene unos tintes marcados de imperialismo. La comunicación para el desarrollo en el enfoque actual se contrapone a esta definición por su carácter situado, abogando por la mejora de bienestar de una población, que debido a la diversidad inherente no se puede producir por medio de modelos universales. Para esto debe pasar de una difusión únicamente, a un dialogo y a la participación de los saberes sociales, por ello, la comunicación se convierte en un derecho y debe democrati- 
zarse (Gumucio y Tufte, 2006). Dentro de estos enfoques actuales se encuentra el desarrollo económico local, donde este viene del potencial endógeno del territorio, es decir, de sus recursos económicos, humanos, institucionales y culturales, de esta manera el territorio aumenta su importancia relativa. Cuando este desarrollo es liderado por la comunidad y toma las dimensiones económicas, socioculturales, políticas y las sostenibilidad se le llama desarrollo económico local endógeno (Vázquez Barquero, 1988). Este tipo de desarrollo permite una integración social y económica y, al mismo tiempo, organiza el territorio "desde abajo" teniendo en cuenta sus particularidades. Las organizaciones deben propender a optimizar los recursos del territorio para generar procesos de crecimiento en una economía de mercado, para lograr economías de escala, aprovechamiento de recursos y generación de innovaciones.

Para esta utilización eficiente del potencial de desarrollo deben existir en las regiones, códigos de conducta, instituciones, mecanismos de regulación económica, organización productiva, tradiciones locales y una estructura social determinada (CEPAL/GTZ, 2000). Esto indica que los sistemas productivos locales son fundamentales en esta dinámica, pues ayudan a realizar una organización sistémica de las unidades de producción, disminuyendo los costos de transacción y generando economías de escala.

El paradigma del desarrollo económico local comparte con el paradigma anterior la necesidad de generar economías de escala, innovaciones y de aprovechar los recursos, en especial la mano de obra excedente de la agricultura en las dinámicas de crecimiento, pero difiere en 4 aspectos, habla de un desarrollo difuso y localizado, cambia la visión de las grandes empresas por la generación de sistemas locales de pequeñas empresas, entiende que las formas de organización social flexibilizan los mercados de trabajo y que la sociedad civil controla los procesos de desarrollo (CEPAL/GTZ, 2000). Así, la difusión de las innovaciones en el territorio depende de las decisiones de los actores, de introducir y desarrollar paradigmas productivos para mejorar las capacidades de aprendizaje y de esta manera, transformar el entorno por medio de la creación y adaptación de tecnologías, es fundamental entonces entender el sistema de relaciones de los actores y su dimensión cognoscitiva.

Por otra parte, el desarrollo sostenible, desde una definición occidental se determinó por la Comisión Mundial sobre Medio Ambiente y Desarrollo (WCED) o Comisión Brundtland, como "el desarrollo que resuelve las necesidades del presente sin comprometer las capacidades de futuras generaciones para resolver sus propias necesidades" (Elliott, 1994, p. 4). Por todo lo anterior, para que el desarrollo se dé y este sea sostenible, es necesario que se atiendan las necesidades de la población, que sea de naturaleza endógena e independiente y que exista una armonía entre la economía, la parte física o de infraestructura del desarrollo y la parte cultural, pero sin comprometer las capacidades de las generaciones futuras.

\subsubsection{El nuevo conocimiento del Diseño como factor de cambio social}

Para que el diseño sea un agente de desarrollo debe existir un cambio de enfoque, debe ser un generador y difusor de innovaciones, de esta manera debe cambiar su resultado de la mera la actividad de diseñar al análisis de contextos específicos y de los actores que se encuentran en estos. Además, debe estar en capacidad de entender aspectos culturales y 
tecnoculturales, como también las preferencias de comunidades locales, para lograr así una cercanía a la mercadotecnia pero con elementos de imagen, de visualización y simbólicos inherentes a la disciplina (Galán, 2011). Coherente con esta visión del diseño, mucho más compleja, se han detectado diferentes enfoques predominantes que hacen que pase de una visión instrumental, o diseño como herramienta.

Para que exista el sentido de un diseño articulado, con una visión sistémica y estratégica, que lo vuelva un facilitador importante en este "plan para la acción" que necesita el desarrollo, se encuentran posturas predominantes como: la innovación social y el diseño, la Innovación guiada por el diseño (design driven innovation), el diseño centrado en las personas o en los usuarios (human - user centered design - design thinking), la gestión estratégica de diseño (design management); de manera que, a continuación se realizará un resumen de cada una de estas posturas a manera de enfoques específicos dentro del desarrollo sostenible.

\subsubsection{La innovación social y el diseño}

Con respecto a los procesos de innovación social, en un estudio del 2006, Manzini mencionó pruebas de evaluación y rediseño continuo en comunidades creativas (2006, p. 5), en ese sentido, uno de los referentes fundamentales es Piere Lévy y la temática de Inteligencia Colectiva. Cómo se recupera el conocimiento entre comunidades, redes que permitan dejar una huella en el conocimiento de manera participativa, ante todo poder expandir la información para que nuevos usuarios opinen acerca de los cambios sociales que vive el hombre en la actualidad. Para Manzini, la idea de bienestar comprende las maneras de ser y de hacer de los individuos para volver a descubrir el entorno que habitamos de una manera más natural y activa en las comunidades de las cuales hacemos parte.

En el plano Latinoamericano, los diseñadores trabajan con objetivos de sostenibilidad con mayor veracidad en el siglo XXI, allí se ubican los entornos virtuales, las redes, los Ecosistemas de creación (Universidad de los Andes, 2015), la cultura y su significado en una región determinada permite el diseño, la creación hacia una capacidad regeneradora en la sociedad. El mundo en que habitan los individuos, la naturaleza, y el conocimiento son proyectados, diseñados en un ejercicio de experimentación y descubrimiento que se alimenta continuamente.

Por otra parte, teóricamente, el maestro cubano Aurelio Horta Mesa ha opinado que "tiempo/espacio en relación con los procesos de creación, tienen como único fin focalizar la atención, así como ubicar el escenario de estas reflexiones en/desde la cultura latinoamericana" (2004, p. 3); para Horta, así como cambian en el transcurso de la historia las herramientas de trabajo, es decir: tecnologías que van evolucionando con el aporte de la ciencia, igualmente se desarrollan sus procesos de adaptación junto a las estructuras sociales, al cabo, modos de producción que definen el discernir del hombre y su relación con el entorno. Es así como se puede entender desde el pensamiento de diseño Latinoamericano, cómo el ser humano puede potencializar su labor creativa con el diseño como parte de las herramientas de comunicación y representación de información que involucra las nuevas dinámicas sociales y participativas del tiempo actual. 


\subsubsection{El Design Driven Innovation}

El Design Driven Innovation (DDI) o Innovación guiada por el diseño, es un término propuesto por Verganti (2008), y consiste en un nuevo tipo de innovación donde el diseñador dota o cambia de significado a los productos u objetos. Según los manuales de Frascati (OCDE, 2003) y Oslo (OCDE, 2005), existen 2 tipos de innovación radical definidos, las innovaciones tecnológicas, como los son aquellas en producto y las innovaciones no tecnológicas, donde se encierran las innovaciones de marketing y organizacionales. En esta visión y en la mayoría de estudios de medición de la innovación, el diseño se considera dentro de las actividades de innovación que son necesarias para el desarrollo de I+D (ACTI). Esto hace que exista una posible relación oculta entre diseño e innovación, que puede ser detectada desde el enfoque del DDI a partir de la propuesta de un nuevo tipo de innovación en significado (Malaver y Vargas, 2012). La importancia de relacionar la experiencia del ser humano por el conocimiento que tiene de los objetos y el uso que se puede dar con un nuevo objetivo. Por otra parte, Verganti afirma que la innovación radical que se realiza desde el diseño es principalmente en significado pues cambia la concepción que se tiene de un producto en un contexto determinado, es decir, a partir de estudios sociales se detectan y se proponen nuevas formas de interactuar con los productos, servicios y objetos. El design driven innovation o innovación radical en significado se diferencia al diseño centrado en el usuario ya que no parte de necesidades, sino de un profundo análisis de las dinámicas sociales que permite al ecosistema de diseño generar propuestas que puedan interesar al usuario, más allá de lo que cree que necesita.

Para las innovaciones radicales en significado es fundamental la existencia de un ecosistema de diseño que funcione como "intérprete" de las dinámicas sociales y pueda proponerle al usuario nuevas formas de interactuar o de concebir los objetos. Existen intérpretes principales o gatekeepers que debido a su experiencia en el entorno son capaces de predecir hacia dónde irá la innovación. En este orden de ideas, el desarrollo desde el design driven innovation, permite no solo predecir sino moldear los significados de los objetos de una sociedad determinada, a partir de las dinámicas detectadas. Esto es fundamental para generar planes de acción que mejoren las condiciones de las comunidades.

\subsubsection{El Diseño centrado en las personas, el diseño centrado en el usuario y el Design thinking (pensamiento de diseño) como herramientas de gestión e innovación del diseño en Latinoamérica}

El Design Thinking o pensamiento de diseño fue desarrollado por el líder de consultora estadounidense Tim Brown (2008), a partir de la idea de que pensar como un diseñador puede transformar cómo se desarrollan, los productos, los procesos y los negocios de las empresas. La capacidad única de los diseñadores de análisis e interpretación de las necesidades de los usuarios es el eje fundamental de este enfoque del diseño. Las características particulares del diseño permiten una innovación centrada en el usuario a diferencia de las ingenierías y el marketing, que se enfocan en aspectos productivos y comerciales, respectivamente, por medio de técnicas de investigación social, el diseño puede articular ambas dimensiones.

Brown afirma que la innovación está impulsada por una compresión de lo que las personas quieren y necesitan en sus vidas, a partir de la observación directa. Para esto creó un 
perfil del "pensador de diseño" que definía las características más importantes que debería tener esta persona que está en la capacidad de interpretar las necesidades de los otros, entre estas se destacan, la empatía, el pensamiento integrador, el optimismo, la necesidad de experimentar y la colaboración.

Además de esto, también planteó una serie de premisas de este tipo de pensamiento (mindsets) que constituyen el espíritu de la metodología: a) empiece por el principio, donde se involucra al equipo de desarrollo desde el inicio de los proyectos, incluso antes de haber definido el rumbo o el objetivo de la intervención; b) adopte un enfoque centrado en las personas, en la medida que la investigación del pensamiento de diseño debe basarse en la conducta, las necesidades y preferencias de los posibles usuarios; c) pruebe desde el principio y con frecuencia, se busca generar un prototipo del proyecto lo más rápido posible, en cada interacción del proceso, de manera que se falle lo más pronto y pueda mejorarse; d) busque ayuda externa, el pensamiento de diseño busca ampliar el ecosistema de innovación a partir de la co-creación con clientes y consumidores.

Por otra parte, desde España, Pardo Kuklinski (2014) ha trabajado el proyecto Outliers School en Iberoamérica, como método de codiseño a partir de un equipo internacional de profesionales trabajando junto a las organizaciones para resolver problemas y crear prototipos de innovación continua, con una metodología propia basada en el Design Thinking. Este equipo viene trabajando hace más de 20 años en la industria de las TIC y en investigación básica y aplicada, haciendo foco en la cultura digital, la comunicación digital, la educación y la gestión del conocimiento (digitalismo.com/outliersschool).

En la era contemporánea la información, el significado y los códigos que genera en diseño en su enfoque comunicativo, ha permitido denominar al receptor o destinatario de los mensajes como usuario, quien se ha integrado cada vez más a los procesos creativos en torno a la productividad en el medio en el cual el diseño se aplica, de manera que el método Outliers School se enfoca en: "Promover un pensamiento de diseñador para no diseñadores que les permita atacar problemas concretos creando innovación con el usuario en el centro del proceso". (Pardo Kuklinski, 2014, p. 215)

Dicho método basado en la estrategia de Design Thinking o pensamiento de diseño a permitido la innovación con respecto a: 1) empatizar con el público objetivo, de manera que los procesos de representación del diseño funcionen para caracterizar los prototipos de servicios y productos; 2) idear en grupos pequeños y con un tiempo limitado de producción, siempre pensando en convertir los problemas del entorno en oportunidades que hagan de la experiencia un consumo como sistema; 3) promover prototipos de rápida ejecución y bajo presupuesto, sin una gran disponibilidad de recursos financieros para potenciar aún más la creatividad; 4) comunicar los elementos esenciales de solución a otros para que puedan comprenderlos, mejorarlos o integrarlos a sus propios procesos; $y$ finalmente 5) promover que el pensamiento de diseño se convierta en política de empresa desde la dirección.

Este tipo de enfoque teórico - práctico que se ha desarrollado en Iberoamérica, ha permitido caracterizar la acción estratégica y la innovación del diseño en contextos regionales entre los años 2013 y 2016 en escenarios sociales denominados como empresas e instituciones, por ejemplo: Instituto Internacional de Ciências Sociais, IICS (Brasil), Universidad de Guadalajara (México), Sistema de Bibliotecas Públicas de Medellín (Colombia), Minis- 
terio TIC (Colombia), Universidad de Buenos Aires (Argentina), Ministerio de Educación de Perú, Parque Explora (Colombia), Festival Internacional de la Imagen (Colombia), Gobierno del Chubut (Argentina), ESAN Graduate School of Business (Perú), entre otras (outliersschool.net). El ejemplo Outliers School fue uno de los antecedentes que permitió recopilar enfoques teóricos del Diseño que propenden hacia el desarrollo sostenible en Latinoamérica y dar paso a implementar el proceso metodológico pertinente a la construcción del estado del arte según la temática propuesta en el presente documento.

El método de pensamiento de diseño ha sido tan exitoso en el ámbito empresarial, que ha ido migrando hacia la innovación social y el trabajo con comunidades, la misma empresa IDEO (2016), en sus últimos cuadernos de metodología ha ampliado la visión del enfoque llamándolo Human Centered Design (HCD) o diseño centrado en las personas, partiendo de la idea de que las comunidades son los verdaderos expertos en sus problemáticas. El proceso de HCD parte de un conjunto de técnicas de investigación etnográfica donde las soluciones se priorizan por medio de tres "lupas" o dimensiones, la de la deseabilidad, que desea o necesita la comunidad, la factibilidad, lo que es técnica y organizacionalmente factible y la viabilidad, que puede ser financieramente viable.

\subsubsection{La gestión estratégica del Diseño}

La gestión de diseño o la gestión estratégica de diseño es un modelo metodológico que concibe al diseño, no desde la concepción de la disciplina al borde de lo artístico, dedicada a la generación de bienes selectos con altos atributos simbólicos, o bienes "de diseño", sino que le da valores propios de la gestión, que podría ser el más armonizado y coherente con lo que los empresarios buscan de la profesión. La gestión se entiende como la dinámica del diseño que estratégicamente ayuda a mejorar la productividad y la competitividad organizacional.

El enfoque de diseño estratégico transforma los sistemas empresariales y se orienta al mercado a través del incremento de la relación marketing-innovación, como una forma de mejorar la calidad del producto y del servicio. "La innovación ayuda a que las compañías sean más competitivas y tengan un mejor posicionamiento en el futuro". Este enfoque particular del diseño puede parecer menos atrayente o glamuroso, pero en realidad, es el que más aporta externalidades capaces de influir positivamente en el desarrollo de las empresas - por ende en la economía - con un impacto social positivo en la productividad. $\mathrm{Al}$ conectar el punto de vista y las prácticas del diseño, la visión estratégica de los negocios y el enfoque de investigación de las ciencias sociales (Boyer, Cook \& Steinberg, 2012), construye una disciplina integradora de conocimiento. Del diseño adopta: a) La visión centrada en el usuario/consumidor; b) los procesos estructurados que conducen hacia la innovación; c) la confianza en la intuición; d) la sensación de comodidad con la incertidumbre y la toma de riesgos; e) el fomento del pensamiento abductivo, el cual visualiza soluciones que aún no existen; f) la naturaleza experimentadora de los diseñadores, quienes aprenden continuamente de sus errores y reiteraciones, como también su capacidad de transformar las ideas en representaciones tangibles (Best, 2011; Boyer, Cook, \& Steinberg, 2012; Brown, 2008; Fraser, 2007; Jensen, 2009; Steinberg, 2013; Stevens, 2009; Tavares de Moura \& Krumholz Adler, 2011; Walters, 2011). Es así como la convergencia disciplinar permite en estos aspectos el trabajo colaborativo. 
Por otra parte, de los negocios asume: 1) el deseo por generar valor a la organización y el énfasis por conocer, aprender y conectar con las distintas partes involucradas (stakeholders); 2) la orientación hacia los objetivos; 3 ) la capacidad de gestionar, organizar, implementar y evaluar proyectos; 4) el entusiasmo por el pensamiento inductivo y deductivo que permite aprender de los patrones del pasado (Best, 2011; Boyer, Cook, \& Steinberg, 2012; Brown, 2008; Euchner, 2012; Lockwood, 2010; Martin, 2007; Van Bergen et al., 2012). Es ense sentido, se enriquece la experiencia del diseño al potencializar el impacto que puede generar en una empresa como escenario social que fomenta el emprendimiento en un sentido económico que aporta al desarrollo de una región.

Así mismo, de la investigación cualitativa promovida por las ciencias sociales, el diseño estratégico: obtiene el interés por empatizar, lo cual permite conocer a profundidad al usuario/consumidor y su contexto; conserva la perseverancia por detectar los problemas claves a resolver y la ambición por conocer cómo se conectan los distintos aspectos de una problemática. (Boyer, Cook, \& Steinberg, 2012; Brown, 2008; Euchner, 2012; Lockwood, 2010) De esta forma, para que el diseñador pueda convertirse en un verdadero agente de desarrollo debe propender a tener un rol estratégico y de mejora de la competitividad en las comunidades empresariales, teniendo en cuenta la relevancia de los sistemas productivos locales en los procesos de desarrollo y el contexto actual donde la globalización, la inestabilidad económica y los constantes cambios tecnológicos generan un entorno altamente variable e inestable para los negocios. (Fraser, 2007; Goktan \& Miles, 2011; Morris, 2011; Nwagbara \& Reid, 2013). Es por esto que el fin último del diseñador es ayudar a desarrollar ventajas competitivas sostenibles y autogestionables en estos sistemas.

\subsection{Desarrollo metodológico: Uso del instrumento bibliométrico}

Siguiendo el tema de estudio presentado anteriormente y para la construcción del estado del arte propio de Latinoamérica, se ubicaron de las palabras clave: a) Innovación Social, b) Gestión estratégica de diseño, c), User centered innovation (innovación centrada por el usuario), d) Co-diseño, E) Co-creación - Diseño, f) Design thinking (pensamiento de diseño), g) Design driven innovation (innovación dirigida por el diseño. Las bases de datos que se consultaron fueron Scielo, ProQuest, Google Scholar, entre otras, ubicando la búsqueda entre el año 2000 hasta el 2016. El estudio bibliométrico se realizó con las siguientes variables. (Ver Figura 1)

Para lograr la búsqueda, fue necesario clasificar verificar en las bases de datos los títulos de los documentos que aparecieron de acuerdo a cada categoría, debido a la amplia cantidad de resultados que presentaban cada una, por ejemplo: en Google Scholar, para la categoría de Co-creación - Diseño, de 530 resultados sólo se identificaron 4 con el enfoque requerido, en Innovación Social - Diseño, de 250 resultados sólo 4 podían sumarse al estudio bibliométrico, en la categoría de Codiseño, de 438 resultados se encontraron 3 y así sucesivamente. 


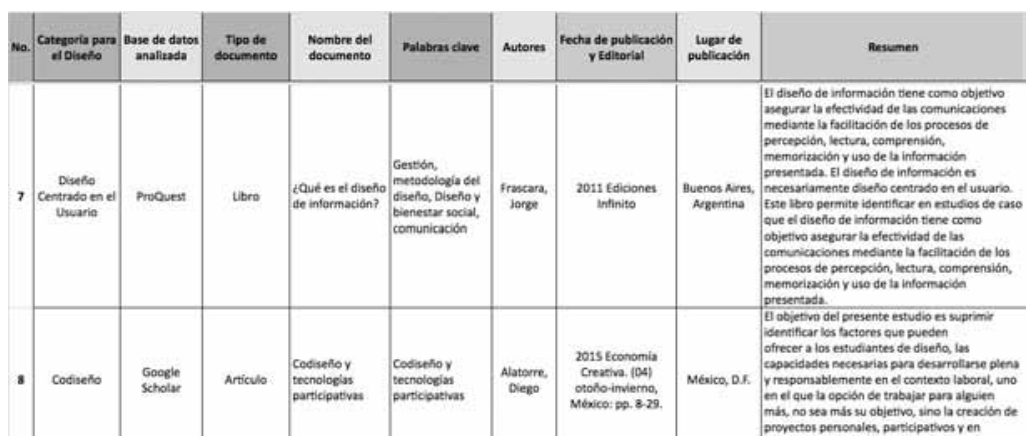

Figura 1. Variables utilizadas en el estudio bibliométrico. Fuente: Autoría propia.

De esta manera, en total se hallaron 52 documentos entre ellos 32 artículos en revistas indexadas, especialmente de universidades latinoamericanas con facultades de humanidades; 9 libros de editoriales especializadas en Diseño, 4 tesis de maestría y 7 informes de investigación y ponencias. A continuación se presenta la Figura 2, una línea de tiempo relacional, en un ejercicio de visualización de datos presentando los 10 países que se identificaron en el estudio bibliométrico según categorías de búsqueda:

Con respecto a las palabras clave, se identificó el factor del Desarrollo Sostenible en cuanto al territorio, la economía regional, la innovación tecnológica unida a la comunicación y al diseño en la sociedad, la responsabilidad en aspectos de aprendizaje en comunidades, la gestión y la metodología del diseño en proyectos en los cuales intervienen usuarios y diseñadores para generar un bienestar social y cultura participativa. Por otra parte, se identificó el concepto de interfaz como forma de comunicación que se apoya en el diseño para interactuar con usuarios a manera de experiencia.

La gestión estratégica fue una de las categorías que más presentó aportes de documentos en cuanto a enfoques teóricos del Diseño, pues se hizo evidente en las palabras clave el uso de redes de conocimiento que involucran las empresas e instituciones como escenarios sociales, en donde converge la disciplina del Diseño y su enfoque sostenible, llevado hacia los factores económicos, políticos, ambientales y culturales que requieren de la innovación. De esta forma, la investigación del diseño se unió a los anteriores aspectos como eje que permite articular la disciplina con la intervención del diseñador en el campo laboral, académico y de responsabilidad social, aumentando en el momento actual el significado de un territorio.

Por otra parte,se observó el bajo porcentaje de documentos en revistas bien rankeadas a nivel Latinoamericano, a continuación se presenta la Figura 3, con la gráfica de la búsqueda realizada según indice $H$ de Google Scholar: 

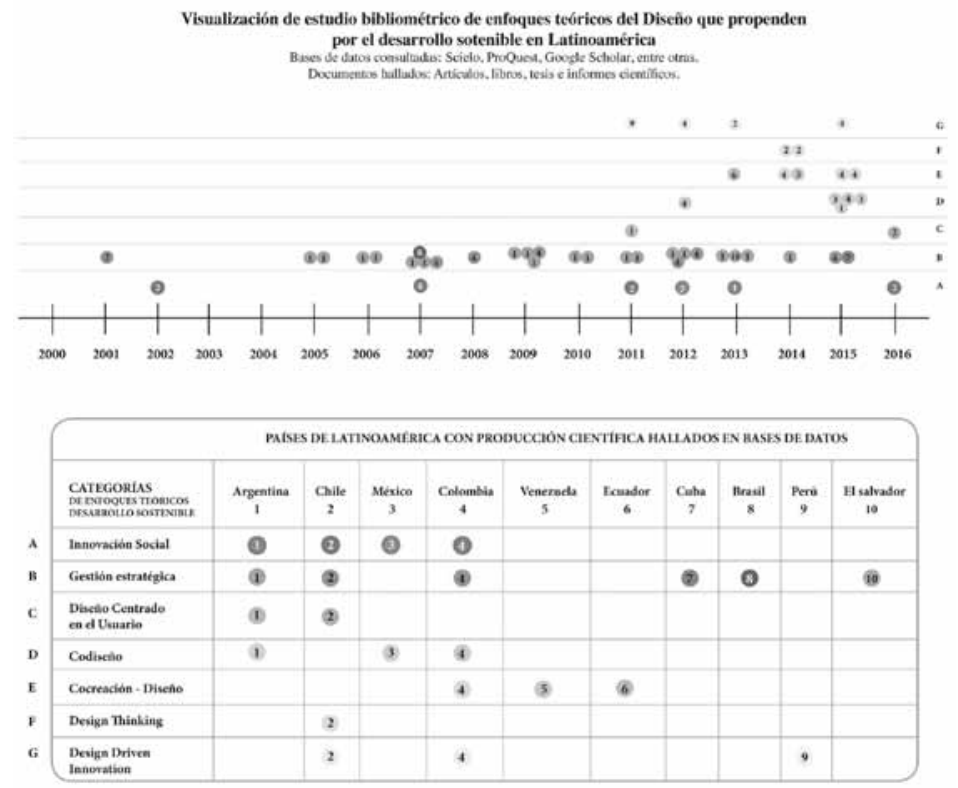

Figura 2.

\begin{tabular}{|c|c|c|}
\hline \multicolumn{3}{|c|}{$\begin{array}{c}\text { Revistas encontradas en el estudio bibliométrico que aparecen } \\
\text { en el índice } \mathrm{H} \text { de Google Scholar }\end{array}$} \\
\hline Publicación & $\begin{array}{l}\text { Cantidad según } \\
\text { fndice H }\end{array}$ & \\
\hline $\begin{array}{l}\text { Revista Innovar } \\
\text { Universidad Nacional de Colombia }\end{array}$ & 9 & 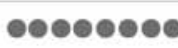 \\
\hline $\begin{array}{l}\text { Revista Desarrollo y sociedad } \\
\text { Buenos Aires, Argentina }\end{array}$ & 7 & 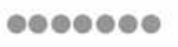 \\
\hline $\begin{array}{l}\text { Revista Ingenieria Industrial } \\
\text { La Habana, Cuba }\end{array}$ & 6 & 사아이 \\
\hline $\begin{array}{l}\text { Revista INTI } \\
\text { Buenos Aires, Argentina }\end{array}$ & 6 & OeOPo \\
\hline $\begin{array}{l}\text { Revista Otra Economia } \\
\text { Brasil }\end{array}$ & 6 & ereede \\
\hline $\begin{array}{l}\text { Revista EURE } \\
\text { Pontificia Universidad Catölica de Chile }\end{array}$ & 4 & 0000 \\
\hline $\begin{array}{l}\text { Revista Sistemas y telemática } \\
\text { Cali, Colombia }\end{array}$ & 3 & 000 \\
\hline $\begin{array}{l}\text { Revista Grafia } \\
\text { Pereira, Colombia }\end{array}$ & 1 & \\
\hline
\end{tabular}

\section{Figura 3.}

Figura 2. Visualización según categorías y países Latinoamericanos hallados en bases de datos frente al Diseño y Desarrollo Sostenible. Fuente: Autoría propia. Figura 3. Búsqueda realizada según índice H de Google Scholar. Fuente: Autoría propia. 


\section{Discusión y conclusiones}

En las visiones contemporáneas de desarrollo sostenible es importante anotar la importancia de entender que cada territorio posee una estructura, sistema productivo, capacidad empresarial y conocimiento tecnológico, recursos naturales e infraestructuras, mercado laboral un sistema social y político, una tradición y cultura, particulares y determinados sobre los que deben construir los procesos de crecimiento económico local. El co-diseño se construye de manera interdisciplinar en escenarios en los que se requiera de la comunicación visual y la participación de los usuarios.

Es un diseño participativo en donde confluyen procesos de interacción, esto hace que se genere una cultura visual al existir retroalimentación como resultado del proceso de comunicación, debido a que el trabajo en equipo genera la alfabetización que permite dar valor a los productos de Diseño Gráfico en el enfoque comunicativo. A continuación se presenta la Tabla 1, con la matriz de identificación de criterios relevantes identificados en el instrumento bibliométrico:

Luego de un análisis de los artículos encontrados en el estudio bibliométrico se denotan varias conclusiones de la temática en Latinoamérica, por ejemplo, la mayoría de documentos se concentran en el periodo de tiempo de 2007-2016, lo que muestra que son relativamente nuevos en la región y algunos son emergentes como el Design Thinking. Por otra parte, la mayoría de aportes que hacen los autores latinoamericanos son desde la parte metodológica y de aplicación de los enfoques, sin embargo no se hacen grandes desarrollos teóricos que cambien la visión de las perspectivas ya analizadas.

La categoría que tiene más documentos y por ende más desarrollo temático es la de gestión estratégica de diseño, los aportes aquí no solo son metodológicos, de estudios de caso y de aplicación, sino que allí confluyen documentos e informes que han caracterizado el aporte económico del diseño en las comunidades productivas. Sumado a lo anterior, gran parte de la producción de documentos sobre gestión de diseño aplicada se encuentra en Argentina donde el desarrollo de instituciones como el INTI (Instituto Nacional de Tecnología Industrial) y el CMD (Centro metropolitano de diseño) han registrado juiciosamente las dinámicas de la disciplina en las comunidades productivas.

Colombia hace un importante aporte teórico al definir las capacidades de diseño en pequeñas y medianas empresas manufactureras, a partir de un estudio relacionar de autores locales e internacionales (Aguilar, Z. J. J \& Hernandez R. D.) caracterizando 4 posibles escenarios de incorporación del diseño en la industria manufacturera 2012, centrado en el experto, centrado en la herramienta, centrado en la estrategia y centrado en los procesos. Todo lo anterior indica que en Latinoamérica se han recibido los cambios relacionados con la temática del desarrollo sostenible como son los factores sociales, económicos, políticos, culturales y ambientales que convergen para construir el momento histórico actual, en donde el diseñador puede innovar como generador de ideas que otorguen bienestar, como lo indica Manzini en cuanto a las maneras de ser y de hacer de los individuos para volver a descubrir el entorno que habitamos de una manera activa en las comunidades de las cuales hacemos parte. 


\begin{tabular}{|c|c|c|c|c|}
\hline Enfoque & $\begin{array}{l}\text { Definición } \\
\text { predominante }\end{array}$ & $\begin{array}{l}\text { Aporte teórico en } \\
\text { Latinoamérica }\end{array}$ & Artículos más relevantes & $\begin{array}{l}\text { Autores - } \\
\text { nacionalidades }\end{array}$ \\
\hline $\begin{array}{l}\text { Innovación } \\
\text { social }\end{array}$ & $\begin{array}{l}\text { - Necesidad de } \\
\text { comunidades } \\
\text { para generar } \\
\text { redes de } \\
\text { conocimiento, } \\
\text { - Inteligencia } \\
\text { colectiva. } \\
\text { - Recuperación } \\
\text { del conocimiento } \\
\text { (Manzini) }\end{array}$ & $\begin{array}{l}\text { Objetivos de } \\
\text { sostenibilidad, entornos } \\
\text { virtuales y ecosistemas } \\
\text { de creación }\end{array}$ & $\begin{array}{l}\text { Innovación, Desarrollo Territorial y } \\
\text { Local, Empresa, Geografía Económica. } \\
2002 \\
\text { Diseño participativo y sustentable } 2013\end{array}$ & $\begin{array}{l}\text { Méndez, R. } \\
\text { Chile } \\
\text { Valdés, S. } \\
\text { Argentina }\end{array}$ \\
\hline $\begin{array}{l}\text { Gestión } \\
\text { estratégica }\end{array}$ & & $\begin{array}{l}\text { Apropiación, registro } \\
\text { e implementación de } \\
\text { metodologías de gestión } \\
\text { estratégica más allá de } \\
\text { la discusión teórica con } \\
\text { resultados tangibles. } \\
\text { Estudios de caso } \\
\text { Definición de las } \\
\text { capacidades de diseño } \\
\text { específicamente en el } \\
\text { sector de las PyMEs }\end{array}$ & $\begin{array}{l}\text { PyMEs, gestión de diseño, política } \\
\text { pública } \\
2007 \\
\text { Hacia un diseño disciplinar inclusivo: } \\
\text { Roles sociales del diseño industrial en } \\
\text { Argentina } 2009 \\
\text { Diseño e innovación para el desarrollo } \\
\text { del capital social. El caso Manos } \\
\text { del Delta. Cooperativa de Artesanos } \\
\text { Isleños. Bs. As. Argentina } \\
2007 \\
\text { Diseño Estratégico: Motor de } \\
\text { innovación y transformación } \\
\text { organizacional } \\
2013 \\
\text { Una interpretación de capacidades } \\
\text { de diseño industrial en pequeñas y } \\
\text { medianas empresas manufactureras } \\
2012\end{array}$ & $\begin{array}{l}\text { Ariza, R. } \\
\text { Argentina } \\
\text { Senar, P. } \\
\text { Argentina } \\
\text { Urquilla, A. El } \\
\text { Salvador } \\
\text { Aguilar, Z.J.J \& } \\
\text { Hernandez } \\
\text { R.D Colombia }\end{array}$ \\
\hline $\begin{array}{l}\text { Codiseño - } \\
\text { co-creación }\end{array}$ & & & $\begin{array}{l}\text { Perspectivas de la co-creación para } \\
\text { el desarrollo de nuevos productos: } \\
\text { Interacción y generación de valor. } 2015 \\
\text { Aproximaciones conceptuales para } \\
\text { entender el Diseño en siglo XXI. } 2015 \\
\text { Co-creando valor público. Desafíos } \\
\text { pendientes para América Latina } \\
2015\end{array}$ & $\begin{array}{l}\text { Giraldo, J.L., \& } \\
\text { Aguilar, Z.J.J } \\
\text { Colombia } \\
\text { Ferruzca, M, } \\
\text { México } \\
\text { Zurbriggen, C } \\
\text { \& González M } \\
\text { Argentina }\end{array}$ \\
\hline $\begin{array}{l}\text { Design } \\
\text { Driven } \\
\text { innovation }\end{array}$ & $\begin{array}{l}\text { El DDI se } \\
\text { centra en la } \\
\text { interpretación de } \\
\text { las comunidades } \\
\text { y el cambio de } \\
\text { significado de } \\
\text { los productos, } \\
\text { comunicaciones } \\
\text { y servicios. } \\
\text { (Verganti) }\end{array}$ & $\begin{array}{l}\text { Construcción de } \\
\text { métodos de medición de } \\
\text { este tipo de innovación } \\
\text { en significado, a partir } \\
\text { del descubrimiento del } \\
\text { vínculo oculto entre } \\
\text { innovación y diseño. }\end{array}$ & $\begin{array}{l}\text { Luces y sombras del vínculo entre el } \\
\text { diseño y la innovación industrial. } 2012\end{array}$ & $\begin{array}{l}\text { Malaver, F } \\
\text { \& Vargas, M } \\
\text { Colombia }\end{array}$ \\
\hline
\end{tabular}

Tabla 1. Matriz de identificación de criterios relevantes en la búsqueda de enfoques teóricos del diseño que propenden por el Desarrollo Sostenible en Latinoamérica. Fuente: Autoría propia. 
Los caminos que surgen a partir del anterior estudio bibliométrico, hacen que se busque la manera de encontrar nuevas bases de datos a partir de redes de conocimiento del diseño sostenible en Latinoamérica, o proponerlas, de manera que la experiencia de docentes de investigación encaminados al diseño, intercambien datos relevantes frente a la innovación social, a las estrategias de co-creación en los escenarios sociales donde se genera sinergia dentro de una región. Esto permitirá que los hallazgos investigativos no sólo aporten a un proyecto en particular, o a una región, sino a dejar una huella en la evolución del conocimiento en nuestro continente, al llevar un enfoque sistémico que propenda por el desarrollo sostenible como dinámica participativa en sectores académicos, empresariales y sociales en general.

\section{Referencias bibliográficas}

Beltrán, L. R. (2006). Rural development and social communication: relationships and strategies. En A. Gumucio-Dragon y T. Tufte (Eds.).Communication for social change anthology: Historical and contemporary readings (pp. 76-87). New Jersey, NJ: Communication for Social Change Consortium.

Bernatene, R. (2007). Programa de diseño Inti. Conferencia presentada en el ciclo de charlas Hablando de Diseño, Instituto Nacional de Tecnología Industrial, Buenos Aires, Argentina.

Best, K. (2011). What can design bring to strategy? Designing thinking as a tool for innovation and change. Rotterdam, Netherlands: Inholland University of Applied Sciences.

Boyer, B., Cook, J. W. \& Steinberg, M. (2012). In studio: Recipes for Systemic Change. Helsinki, Finland: Sitra - Helsinki Design Lab.

Brown, T. (2008). Design thinking. Harvard Business Review, (June 2008), 84-92.

CEPAL/GTZ (2009). Desarrollo económico local y descentralización: aproximación a un marco conceptual, CEPAL, Santiago de Chile.

Dissanayake, W. (2006). A Buddhist approach to development: a Sri Lankan endeavor. En A. Gumucio-Dragon y T. Tufte (Eds.).Communication for social change anthology: Historical and contemporary readings (pp. 243-245). New Jersey: Communication for Social Change Consortium.

Durán Bobadilla, L. (2015). "Signos individuales en la experiencia de la Comunicación Visual”. Tesis de Maestría en Diseño y Creación Interactiva de la Universidad de Caldas. Manizales, Colombia. Recuperado de: http://www.maestriaendiseno.com/tesis/

Elliot, J. (1994). An Introduction to Sustainable Development, London: Routledge, pp. 4-5. Euchner, J. (2012). Design thinking: An interview with Roger Martin. Research Technology Management, 55(3), 10-14.

Frascara, J. (2006). El diseño de comunicación. Buenos Aires: Ediciones Infinito.

Fraser, H. M. (2007). The practice of breakthrough strategies by design. Journal of Business Strategy, 28(4), 66-74.

Galán, B. (2011). Empresas creativas y conocimientos intangibles. En Instituto Metropolitano de diseño e innovación (IMDI) (Ed.). Aportes de diseño, una herramienta para mejorar el desempeño empresarial (pp. 25-36). Buenos Aires: Centro Metropolitano de Diseño. 
González Ruiz, G. (1994). Estudio de Diseño. Emecé Editores, Buenos Aires, Argentina.

Goktan, A. B. \& Miles, G. (2011). Innovation speed and radicalness: Are they inversely related? Management Decision, 49(4), 533-547.

Gumucio, A. y Tufte, T. (Eds.) (2006). Communication for Social Change Anthology: Historical and Contemporary Readings. Nueva York, NJ: Communication for Social Change Consortium.

Horta Mesa, A. (2004). "Pensar el diseño..., hacer la síntesis", en Pensar el Diseño. Una profesión del Siglo XXI. Universidad Veritas. San José, Costa Rica.

IDEO (2015). The field guide to human-centered design: Design kit. San Francisco: IDEO.

Jensen, A. K. (2009). Strategic design: Achieving utopian goals. Mind Design, 16. Obtenido de http://www.dcdr.dk/uk/menu/update/webzine/articles/strategic-design-achievingutopiangoals

http://www.dcdr.dk/uk/menu/update/webzine/articles/strategic-design-achieving-uto piangoals

Lockwood, T. (2010). Transition: How to become a more design-minded organization. DMI News \& Views, 21(2), 29-37.

Manzini, E. (2006). "Design, ethics and sustainability“, [en línea], disponible en: http://design blog.uniandes.edu.co/blogs/desis/files/2009/06/060828-design-ethics-sustainability.pdf.

Manzini, E. (2009). “New Design Knowledge” en Design Studies, vol 30, núm. 1, pp. 4/12

Martin, R. (2007). Design and business: Why can't we be friends? Journal of Business Strategy, 28(4), 6-12.

Malaver, F. \& Vargas, M. (2012). Luces y sombras del vínculo entre el diseño y la innovación industrial. Innovar, 22(46), 149-164.

Nwagbara, U. \& Reid, P. (2013). Corporate Social Responsibility (CSR) and management trends: Changing times and changing strategies. Economic Insights - Trends and Challenges, 2, 12-19.

OCDE (Organisation for Economic Co-operation and Development), \& Fundación Española para la Ciencia y la Tecnología. (2003). Manual de Frascati 2002: Propuesta de norma práctica para encuestas de investigación y desarrollo experimental: medición de las actividades científicas y tecnológicas. Madrid: Fundación Española para la Ciencia y la Tecnología, publicado por acuerdo con la OCDE.

OCDE/European Communities. (2005). Manual de Oslo. Guía para la recogida e interpretación de datos sobre innovación (3a. ed.). Madrid, España: Grupo Tragsa.

Outliers School (2016). Cultura digital, educación, evolución de medios, gestión de conocimiento. Recuperado de: http://outliersschool.net/

Pardo Kuklinski, H. (2014). Opportunity Valley. Lecciones <aún> no aprendidas de treinta años de contracultura digital. Outliers School. Barcelona. Recuperado de: www.oppor tunityvalley.net

Steinberg, M. (2013). Strategic design. Conferencia presentada en el Simposio RE: Look Design - Core Competencies in Transition. Konstfack, University College of Arts, Crafts and Design, Stockholm, Sweden.

Stevens, J.S. (2009). Design as a strategic resource: Design's contributions to competitive advantage aligned with strategy models. Tesis doctoral. University of Cambridge, Cambridge, UK. 
Tavares de Moura, H. \& Krumholz Adler, I. (2011). The ecology of innovation and the role of strategic design. Strategic Design Research Journal, 4(3), 112-117.

TED Conferences, LLC. (2013). TEDxRioDeLaPlata. Nunca pidas permiso. Recuperado el 14 de junio de 2016 de http://tedxtalks.ted.com/video/Nunca-pidas-permiso-Ronald-Shak Uribe Orozco, M. (2009). "Caracterización del objeto de estudio desde diseño de comunicación (gráfico / visual) y su relación con las diferentes dimensiones de aplicación: empírica, profesional y académica". Tesis de Maestría en Diseño y Creación Interactiva de la Universidad de Caldas. Manizales, Colombia. Recuperado de: http://www.new.maestriaendiseno.com/ pdf/01MarioFernandoUribe.pdf

Universidad de los Andes (2015). Ecosistemas de creación. Facultad de Arquitectura y Diseño. Recuperado de http://arqdis.uniandes.edu.co/?uniandes_proyectos=ecosistemasculturales-from-economies-to-ecologies-of-creation

Universidad de Palermo (2015) Comité del Diseño Latino - Embajadores del Diseño Latino. Facultad de Diseño y Comunicación. Buenos aires, Argentina. Recuperado de: http:// www.palermo.edu/dyc/embajadores_diseno_latino/embajadores/joaquim_redig.html

Walters, H. (2011). The seven deadly sins that choke out innovation. Fast Company Co.Design. Obtenido de http://www.fastcodesign.com/1663192/theseven-deadly-sins-that-chokeout-innovation

Van Bergen, E., Gokgoz, E., Singh, G., Martin, J. D., Ferreira de Sá, M. \& Melgarejo, M.]. (2012). Design the new business [Video]. Obtenido de http://www.designthenewbusi ness.com

Vázquez Barquero, A. (1988), Small-scale industry in rural areas: The Spanish experience since the beguinning of this century, en K.J. Arrow (ed.) The Balance between Industry and Agriculture in Economic Development. Mcmillan, London.

Verganti, R. (2008). Design, Meanings, and Radical Innovation: A Metamodel and a Research Agenda. Journal of Product Innovation Management, 25(5), 436-456.

\begin{abstract}
This work presents a bibliometric study of scientific documents such as papers, books, thesis and research reports, in which predominant theoretical approaches on design as a discipline published in Latin American indexed journals from 2000 to 2016, using quantitative methodology to locate in databases like Scielo, ProQuest, Google Scholar, among other. It seeks to build a review of the prevailing approaches in this period that are related to the impact of design in the development of communities. They were taken as a basis categories: design thinking, user-centered design, co-creation and strategic design management (more emerging). It concludes with a comparative table, summarizing these approaches so that identifies history against the subject of study, which contribute to research projects in the current historical moment.
\end{abstract}

Key words: Social innovation - sustainable development - co-creation - strategic design management - territory. 
Resumo: O texto apresenta um estudo bibliométrico de documentos científicos (artigo, livro, tese e relatório de pesquisa), a partir dos quais se encontram os principais abordagens teóricas do design, publicados em revistas indexadas da América Latina, entre 2000 e 2016. O artigo busca construir uma revisão da perspectiva prevalecente relacionada com o impacto da disciplina em o desenvolvimento das comunidades; e foi feito com base nas categorias: o pensamento de design, design focado em pessoas-usuários, co-criação e gestão de design estratégico (além das emergente). Conclui-se com um quadro comparativo resumindo estas abordagens, a fim de identificar antecedentes, que se constituem como contribuições para projetos de pesquisa atual e futuro.

Palavras chave: inovação social - desenvolvimento sustentável - co-criação - gestão de design estratégico - território. 